\title{
Microarray data analysis on gene and miRNA expression to identify biomarkers in non-small cell lung cancer
}

\author{
Xiang Jin', Yinghui Guan ${ }^{1 *}$, Zhen Zhang ${ }^{2}$ and Hongyue Wang ${ }^{3}$
}

\begin{abstract}
Background: The aim of this study was to gain further investigation of non-small cell lung cancer (NSCLC) tumorigenesis and identify biomarkers for clinical management of patients through comprehensive bioinformatics analysis.

Methods: miRNA and mRNA microarray datasets were downloaded from GEO (Gene Expression Omnibus) database under the accession number GSE102286 and GSE101929, respectively. Genes and miRNAs with differential expression were identified in NSCLC samples compared with controls, respectively. The interaction between differentially expressed genes (DEGs) and differentially expressed miRNAs (DEmiRs) was predicted, followed by functional enrichment analysis, and construction of miRNA-gene regulatory network, protein-protein interaction (PPI) network, and competing endogenous RNA (ceRNA) network. Through comprehensive bioinformatics analysis, we anticipate to find novel therapeutic targets and biomarkers for NSCLC.
\end{abstract}

Results: A total of 123 DEmiRs (5 up- and 118 down-regulated miRNAs) and 924 DEGs (309 up- and 615 down-regulated genes) were identified. These genes and miRNAs were significantly involved in different pathways including adherens junction, relaxin signaling pathway, and axon guidance. Furthermore, hsa-miR-9-5p, has-miR-196a-5p and hsa-miR-31-5p, as well as hsa-miR-1, hsa-miR-218-5p and hsa-miR-135a-5p were shown to have higher degree in the miRNA-gene regulatory network and ceRNA network, respectively. Furthermore, BIRC5 and FGF2, as well as RTKN2 and SLIT3 were hubs in the PPI network and ceRNA network, respectively.

Conclusion: Several pathways (adherens junction, relaxin signaling pathway, and axon guidance) miRNAs (hsa-miR-9-5p, has-miR-196a-5p, hsa-miR-31-5p, hsa-miR-1, hsa-miR-218-5p and hsa-miR-135a-5p) and genes (BIRC5, FGF2, RTKN2 and SLIT3) may play important roles in the pathogenesis of NSCLC.

Keywords: Non-small cell lung cancer, Differentially expressed genes, miRNA, Regulatory network, Microarray data analysis

\section{Background}

Lung cancer presents the major cause of cancer deaths in the past few decades and has been a major public health problem [1]. Histologically, non-small cell lung cancer (NSCLC) accounts for more than $80 \%$ of lung malignancy

\footnotetext{
* Correspondence: Yinny HG@126.com

'Department of Respiration, The First Hospital of Jilin University, No. 1 Xinminda Street, Changchun 130021, China

Full list of author information is available at the end of the article
}

cases, with an overall 5-year survival rate below 15\% [2]. More than $70 \%$ of these cases are diagnosed with locally advanced or metastatic disease and the prognosis for NSCLC patients remains poor [3]. Therefore, it places a high priority on elucidating the molecular mechanisms of NSCLC pathogenesis and identify diagnostic or predictive biomarkers.

Research has revealed significant interactions between gene alterations and tumorigenesis and tumor progression of many types of cancers [4]. CD44 was shown to

(c) The Author(s). 2020 Open Access This article is licensed under a Creative Commons Attribution 4.0 International License, which permits use, sharing, adaptation, distribution and reproduction in any medium or format, as long as you give appropriate credit to the original author(s) and the source, provide a link to the Creative Commons licence, and indicate if changes were made. The images or other third party material in this article are included in the article's Creative Commons licence, unless indicated otherwise in a credit line to the material. If material is not included in the article's Creative Commons licence and your intended use is not permitted by statutory regulation or exceeds the permitted use, you will need to obtain permission directly from the copyright holder. To view a copy of this licence, visit http://creativecommons.org/licenses/by/4.0/ The Creative Commons Public Domain Dedication waiver (http://creativecommons.org/publicdomain/zero/1.0/) applies to the data made available in this article, unless otherwise stated in a credit line to the data. 
be overexpressed in NSCLC and involved in the occurrence and migration of NSCLC [5]. The genes L1TD1 (LINE-1 Type Transposase Domain Containing 1) and SPAG6 (Sperm Associated Antigen 6) are tumor-specifically methylated in NSCLC [6]. Recently, Morris et al. reported that FPR1 mRNA levels in whole blood predicts both NSCLC and small cell lung cancer [7]. MicroRNAs (miRNAs) are a large group of small non-coding RNAs of 20-24 nucleotides that are involved in the fine-tuning of various biological processes. They bind to multiple target mRNAs typically in the 3 '-untranslated region ( $3^{\prime}$-UTR) and govern gene expression at the post-transcriptional level [8]. A possible tumor suppressor role for hsa-miR-30d in progression of NSCLC was shown in a recent study [9]. Yang et al. demonstrated that miR-598 suppressed the invasion and migration in NSCLC as a tumor suppressor through negatively regulate Derlin-1 (DERL1) and epithelial-mesenchymal transition (EMT) [10]. However, the roles of genes and miRNAs in NSCLCs are still not well understood [11].

Expression profiling with high-throughput microarrays has become a mature and widely used technology to obtain more global views on cancer genes and to identify novel cancer biomarkers [12]. In this study, we reported an integrated analyses of miRNAs and gene expression by reanalyzing public datasets from GSE102286 and GSE101929. Differentially expressed genes (DEGs) and differentially expressed miRNAs (DEmiRs) were identified in NSCLC samples compared with controls, respectively. The interaction between DEmiRs and DEGs was predicted, followed by functional enrichment analysis, and construction of miRNA-gene regulatory network, protein-protein interaction (PPI) network, and competing endogenous RNA (ceRNA) network. Through comprehensive bioinformatics analysis, we anticipate to find novel therapeutic targets and biomarkers for NSCLC.

\section{Methods}

\section{Source of the microarray data}

The public Gene Expression Omnibus (GEO) repository at http://www.ncbi.nlm.nih.gov/geo/ serve as a public data archive that freely disseminates high-throughput functional genomic data [13]. In this study, miRNA and mRNA microarray datasets were downloaded from the GEO database under the accession number GSE102286 and GSE101929,
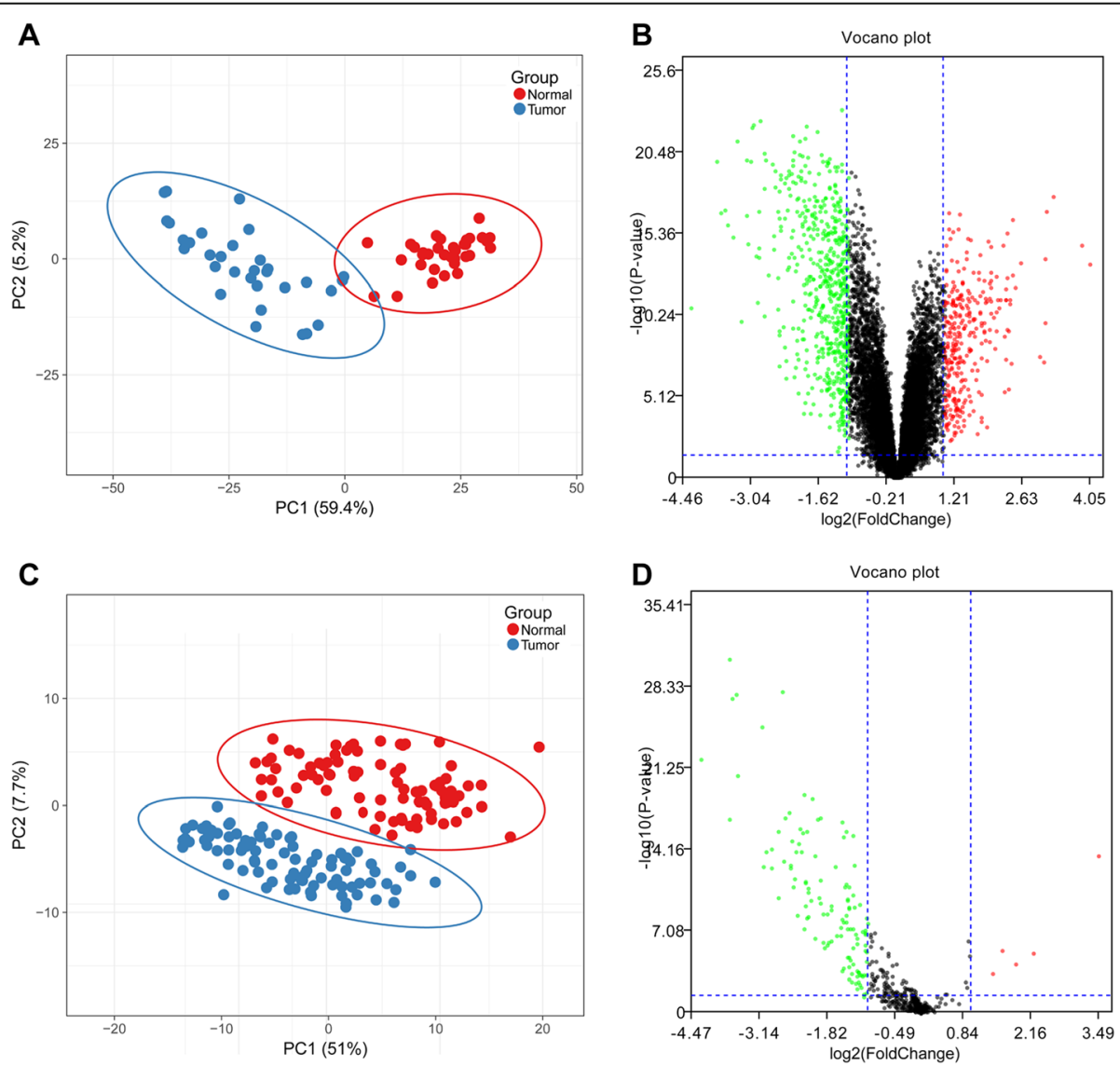

Fig. 1 Principal component analysis (PCA) and volcano plots of differentially expressed genes (DEGs) and differentially expressed miRNAs (DEmiR). a, PCA of DEGs. $\mathbf{b}$, volcano plot of DEGs. c, PCA of DEmiR. $\mathbf{d}$, volcano plot of DEmiR 
respectively. The miRNA dataset GSE102286 included 88 normal control samples and 91 NSCLC samples. All samples were tested using the GPL23871 NanoStringnCounter Human miRNA Expression Assay v1.6 platform. The gene expression profile GSE101929 included 34 normal control samples and 32 NSCLC samples. The platform used for the gene microarray was GPL570 [HG-U133_Plus_2] Affymetrix Human Genome U133 Plus 2.0 Array.

Patients that underwent curative NSCLC surgery between 1998 and 2014 were enrolled. The mRNA and miRNA cohorts included matched tumor and normal pairs. The mRNA cohort included 25 matched pairs and 7 unmatched pairs and the miRNA cohort included 93 matched pairs and two unmatched pairs.

\section{Data preprocessing and DEmiRs and DEGs screening}

The miRNA raw data (RCC files) were received and read into the R statistical environment, followed by data preprocessing using the NanoStringNorm package [14] (v1.2.1, https://cran.r-project.org/web/packages/NanoStringNorm/ ), including background correction, normalization, and concentration prediction. The results of non-human miRNA probes on the chip were removed, and the remnant was used as the final miRNA expression value.

In addition, the mRNA raw data (CEL files) and the platform annotation file were downloaded. The affy package [2] was used for data preprocessing, including background correction, normalization, and expression level calculation. In accordance with the platform annotation
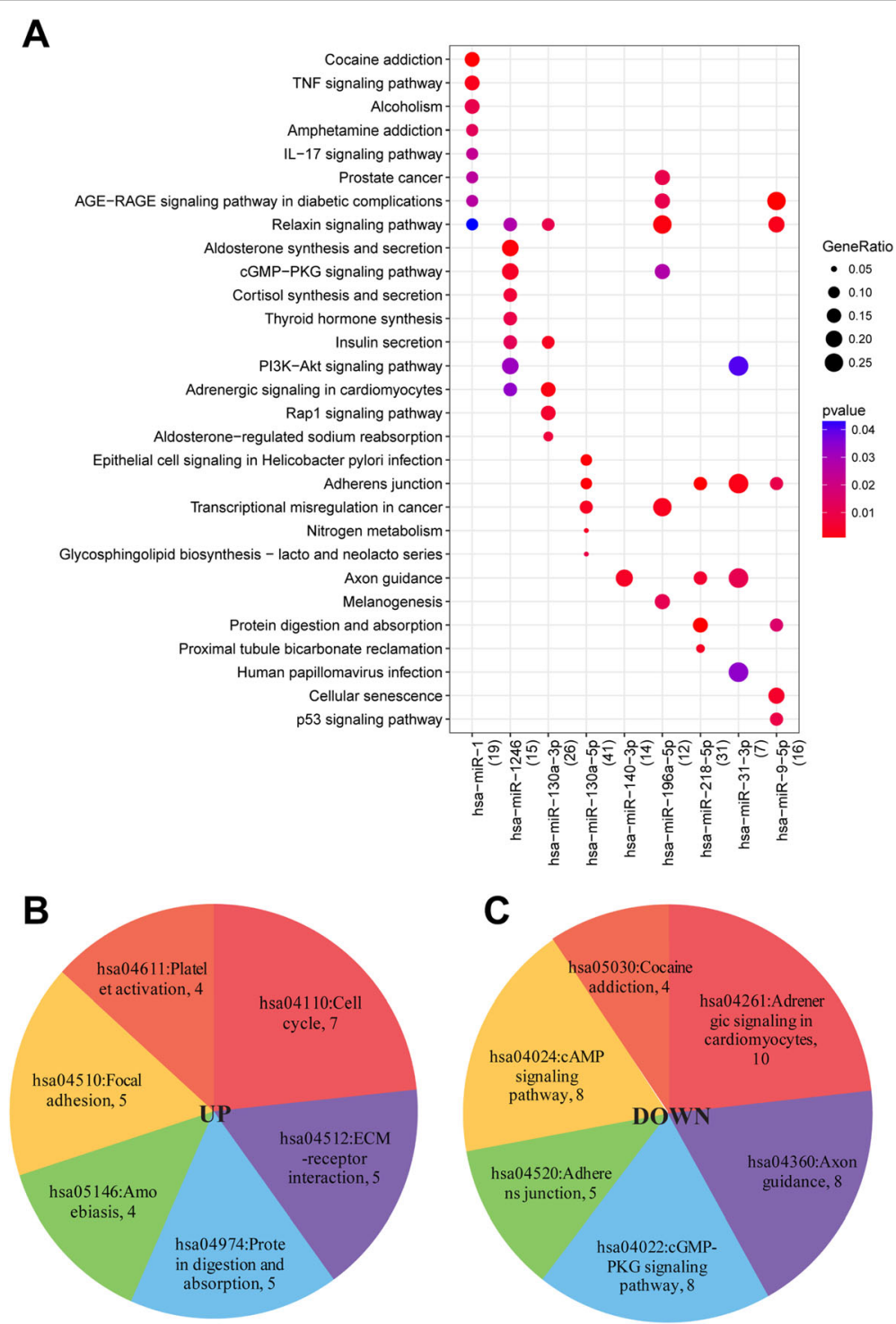

Fig. 2 Kyoto Encyclopedia of Genes and Genomes (KEGG) pathways enriched by miRNAs or genes involved in DEmiR-DEGs. a, KEGG pathways enriched by 8 miRNAs of DEmiR-DEGs. $\mathbf{b}$, KEGG pathway enriched by the up-regulated genes. $\mathbf{c}$, KEGG pathway enriched by down-regulated genes 
Table 1 The enriched pathways by differentially expressed miRNAs

\begin{tabular}{|c|c|c|c|c|}
\hline miRNA & ID & Pathway & $P$ value & Count \\
\hline \multirow[t]{9}{*}{ hsa-miR-9-5p } & hsa04933 & AGE-RAGE signaling pathway in diabetic complications & 4.84E-05 & 4 \\
\hline & hsa04926 & Relaxin signaling pathway & $2.51 \mathrm{E}-03$ & 3 \\
\hline & hsa04218 & Cellular senescence & 4.51E-03 & 3 \\
\hline & hsa04115 & p53 signaling pathway & $9.18 \mathrm{E}-03$ & 2 \\
\hline & hsa04520 & Adherens junction & 1.03E-02 & 2 \\
\hline & hsa04350 & TGF-beta signaling pathway & $1.38 \mathrm{E}-02$ & 2 \\
\hline & hsa04974 & Protein digestion and absorption & 1.57E-02 & 2 \\
\hline & hsa05146 & Amoebiasis & 1.77E-02 & 2 \\
\hline & hsa05142 & Chagas disease (American trypanosomiasis) & 1.99E-02 & 2 \\
\hline \multirow[t]{4}{*}{ hsa-miR-31-3p } & hsa04520 & Adherens junction & 1.90E-03 & 2 \\
\hline & hsa04360 & Axon guidance & $1.08 \mathrm{E}-02$ & 2 \\
\hline & hsa05165 & Human papillomavirus infection & 3.37E-02 & 2 \\
\hline & hsa04151 & PI3K-Akt signaling pathway & 4.04E-02 & 2 \\
\hline \multirow[t]{4}{*}{ hsa-miR-218-5p } & hsa04974 & Protein digestion and absorption & 3.14E-05 & 5 \\
\hline & hsa04520 & Adherens junction & 2.12E-04 & 4 \\
\hline & hsa04964 & Proximal tubule bicarbonate reclamation & 4.06E-03 & 2 \\
\hline & hsa04360 & Axon guidance & 5.77E-03 & 4 \\
\hline \multirow[t]{7}{*}{ hsa-miR-196a-5p } & hsa04926 & Relaxin signaling pathway & 1.04E-03 & 3 \\
\hline & hsa05202 & Transcriptional misregulation in cancer & 2.90E-03 & 3 \\
\hline & hsa05215 & Prostate cancer & 1.03E-02 & 2 \\
\hline & hsa04933 & AGE-RAGE signaling pathway in diabetic complications & 1.07E-02 & 2 \\
\hline & hsa04916 & Melanogenesis & 1.11E-02 & 2 \\
\hline & hsa04022 & cGMP-PKG signaling pathway & 2.75E-02 & 2 \\
\hline & hsa04020 & Calcium signaling pathway & 3.37E-02 & 2 \\
\hline hsa-miR-140-3p & hsa04360 & Axon guidance & $3.90 \mathrm{E}-03$ & 3 \\
\hline \multirow[t]{6}{*}{ hsa-miR-130a-5p } & hsa05120 & Epithelial cell signaling in Helicobacter pylori infection & $5.11 \mathrm{E}-04$ & 4 \\
\hline & hsa04520 & Adherens junction & 6.35E-04 & 4 \\
\hline & hsa05202 & Transcriptional misregulation in cancer & $3.41 \mathrm{E}-03$ & 5 \\
\hline & hsa00910 & Nitrogen metabolism & 3.86E-03 & 2 \\
\hline & hsa00601 & Glycosphingolipid biosynthesis - lacto and neolacto series & $9.62 \mathrm{E}-03$ & 2 \\
\hline & hsa04666 & Fc gamma R-mediated phagocytosis & 1.37E-02 & 3 \\
\hline \multirow[t]{6}{*}{ hsa-miR-130a-3p } & hsa04261 & Adrenergic signaling in cardiomyocytes & 1.47E-03 & 4 \\
\hline & hsa04911 & Insulin secretion & $3.14 \mathrm{E}-03$ & 3 \\
\hline & hsa04015 & Rap1 signaling pathway & $5.38 \mathrm{E}-03$ & 4 \\
\hline & hsa04960 & Aldosterone-regulated sodium reabsorption & 7.33E-03 & 2 \\
\hline & hsa04926 & Relaxin signaling pathway & $1.02 \mathrm{E}-02$ & 3 \\
\hline & hsa04961 & Endocrine and other factor-regulated calcium reabsorption & 1.16E-02 & 2 \\
\hline \multirow[t]{7}{*}{ hsa-miR-1246 } & hsa04925 & Aldosterone synthesis and secretion & 8.59E-04 & 3 \\
\hline & hsa04022 & cGMP-PKG signaling pathway & $3.92 \mathrm{E}-03$ & 3 \\
\hline & hsa04927 & Cortisol synthesis and secretion & 6.97E-03 & 2 \\
\hline & hsa04918 & Thyroid hormone synthesis & $9.52 \mathrm{E}-03$ & 2 \\
\hline & hsa04911 & Insulin secretion & $1.24 \mathrm{E}-02$ & 2 \\
\hline & hsa04922 & Glucagon signaling pathway & 1.79E-02 & 2 \\
\hline & hsa04928 & Parathyroid hormone synthesis, secretion and action & 1.89E-02 & 2 \\
\hline
\end{tabular}


Table 1 The enriched pathways by differentially expressed miRNAs (Continued)

\begin{tabular}{|c|c|c|c|c|}
\hline miRNA & ID & Pathway & $P$ value & Count \\
\hline & hsa04725 & Cholinergic synapse & 2.09E-02 & 2 \\
\hline & hsa04724 & Glutamatergic synapse & 2.17E-02 & 2 \\
\hline & hsa04926 & Relaxin signaling pathway & 2.77E-02 & 2 \\
\hline & hsa04728 & Dopaminergic synapse & 2.81E-02 & 2 \\
\hline & hsa04915 & Estrogen signaling pathway & 3.05E-02 & 2 \\
\hline & hsa04151 & PI3K-Akt signaling pathway & 3.17E-02 & 3 \\
\hline & hsa04261 & Adrenergic signaling in cardiomyocytes & 3.34E-02 & 2 \\
\hline & hsa04310 & Wnt signaling pathway & 3.43E-02 & 2 \\
\hline & hsa04934 & Cushing's syndrome & $3.78 \mathrm{E}-02$ & 2 \\
\hline \multirow[t]{13}{*}{ hsa-miR-1 } & hsa05030 & Cocaine addiction & 2.45E-04 & 3 \\
\hline & hsa04668 & TNF signaling pathway & 2.47E-03 & 3 \\
\hline & hsa05034 & Alcoholism & $1.03 \mathrm{E}-02$ & 3 \\
\hline & hsa05031 & Amphetamine addiction & 1.29E-02 & 2 \\
\hline & hsa04657 & IL-17 signaling pathway & $2.32 \mathrm{E}-02$ & 2 \\
\hline & hsa05215 & Prostate cancer & 2.51E-02 & 2 \\
\hline & hsa04933 & AGE-RAGE signaling pathway in diabetic complications & 2.61E-02 & 2 \\
\hline & hsa04922 & Glucagon signaling pathway & 2.81E-02 & 2 \\
\hline & hsa04060 & Cytokine-cytokine receptor interaction & $3.02 \mathrm{E}-02$ & 3 \\
\hline & hsa04152 & AMPK signaling pathway & $3.78 \mathrm{E}-02$ & 2 \\
\hline & hsa04926 & Relaxin signaling pathway & 4.31E-02 & 2 \\
\hline & hsa04550 & Signaling pathways regulating pluripotency of stem cells & 4.86E-02 & 2 \\
\hline & hsa05418 & Fluid shear stress and atherosclerosis & 4.86E-02 & 2 \\
\hline
\end{tabular}

information, the probes were converted into gene symbols. For multiple probes corresponding to the same gene symbol, the probe average was taken as the final expression value of gene.

The expression matrix was divided into tumor group and normal group, and DEmiRs and DEGs were screened separately. Non-paired t-test provided by limma [15] were used to calculate the $P$-values of significant gene expression differences. Benjamini-Hochberg (BH)-corrected $P$-values $<0.05$ and $\mid \log _{2}$ fold change $(\mathrm{FC}) \mid>1$ were chosen as the threshold for the identification of significant DEmiRs and DEGs.

\section{Identification of DEmiR-DEGs relationship pairs}

miRWalk 2.0 tools [16] was applied to predict the target genes of the top 10 up- and down-regulated miRNAs with high FC value. The miRNA-target gene regulatory data from at least 5 of the 7commonly used databases (miRWalk, miRanda, miRDB, miRMap, miRNAMap, RNA22, Targetscan) were collected. The obtained miRNA-target relationship was matched with DEGs obtained by microarray analysis to obtain the interaction between DEmiRs and DEGs (DEmiR-DEGs relationship pairs).

\section{Pathway enrichment analysis of miRNAs and genes}

The Kyoto Encyclopedia of Genes and Genomes (KEGG) [17] function enrichment analysis of miRNAs involved in the DEmiR-DEG interaction was carried out using R clusterprofiler package [18], and the pathways with $P$-value $<0.05$ and count $\geq 2$ were significantly enriched.

KEGG [17] pathway enrichment analysis was also conducted on the up-and down-regulated genes involved in the DEmiR-DEG relationship pairs. The commonly used DAVID [7] (version6.8, https://davidd.ncifcrf.gov/) was applied for the function enrichment analysis with gene count $\geq 2$ and $P$-value $<0.05$ as the thresholds.

\section{Construction of miRNA-gene regulatory network and module analysis}

Based on the interaction information of the DEmiRDEG, the construction of miRNA-gene regulatory network was performed using the Cytoscape software (vesrion: 3.2.0) [19]. The topological properties of network nodes were analyzed, such as the centrality of nodes. 
PPI network of the DEGs involved in DEMIR-DEG

The STRING database [20] (version: 10.0) was used to predict the PPI pairs of proteins encoded by DEGs involved in the DEmiR-DEG. The input gene set was DEGs and the species was Homo sapiens. The parameter of PPI score was set as 0.4 (indicating medium confidence) [21]. A PPI network involved in DEmiR-DEG was constructed by Cytoscape software (version: 3.2.0) [19].

\section{Construction of ceRNA network}

ceRNA can act as decoys for miRNA binding and form complex regulatory networks based on an "miRNA response element (MRE) language" that regulate the abundance of any MRE-containing RNA transcripts such as long noncoding RNAs (lncRNAs) [22]. We used the miRNA-lncRNA interaction pairs in the starBase database (http://starbase.sysu.edu.cn/) [23] to screen the lncRNAs that have interaction with the DEMIR. The screening criteria for screening miRNA-lncRNA regulatory relationship pairs was medium stringency $\geq 2$ and number of cancer types $\geq 1$. Baes on the predicted miRNA-lncRNA relationship pairs and DEmiR-DEG (miRNA-mRNA) regulatory relationships obtained above, the IncRNA-miRNA-mRNA network (ceRNA network) was constructed using Cytoscape software.

\section{Results}

Identification of DEGs and DEmiRs

After data preprocessing, a total of 924 DEGs were obtained, of which 309 genes were significantly up-regulated and 615 were significantly down-regulated. The principal component analysis (PCA) and volcano plots of DEGs were shown in Fig. 1a and b. Additionally, 123 DEmiRs were identified, among which 5 miRNAs were significantly up-regulated and 118 miRNAs were significantly downregulated. PCA and volcano plots of those DEmiRs were shown in Fig. 1c and d.

Then, by searching the miRWalk2.0 database, we found target genes for 18 mature miRNAs. Finally, we obtained 715 pairs of DEmiR-DEG, including 6 up- and 12 down-regulated miRNAs, as well as 101 up- and 245 down-regulated genes.

\section{Functional enrichment analysis of DEmiRs and DEGs}

Totally, 66 KEGG pathways were enriched by 8 miRNAs of DEmiR-DEG (Fig. 2a and Table 1). Among these, adherens junction, relaxin signaling pathway, axon guidance,

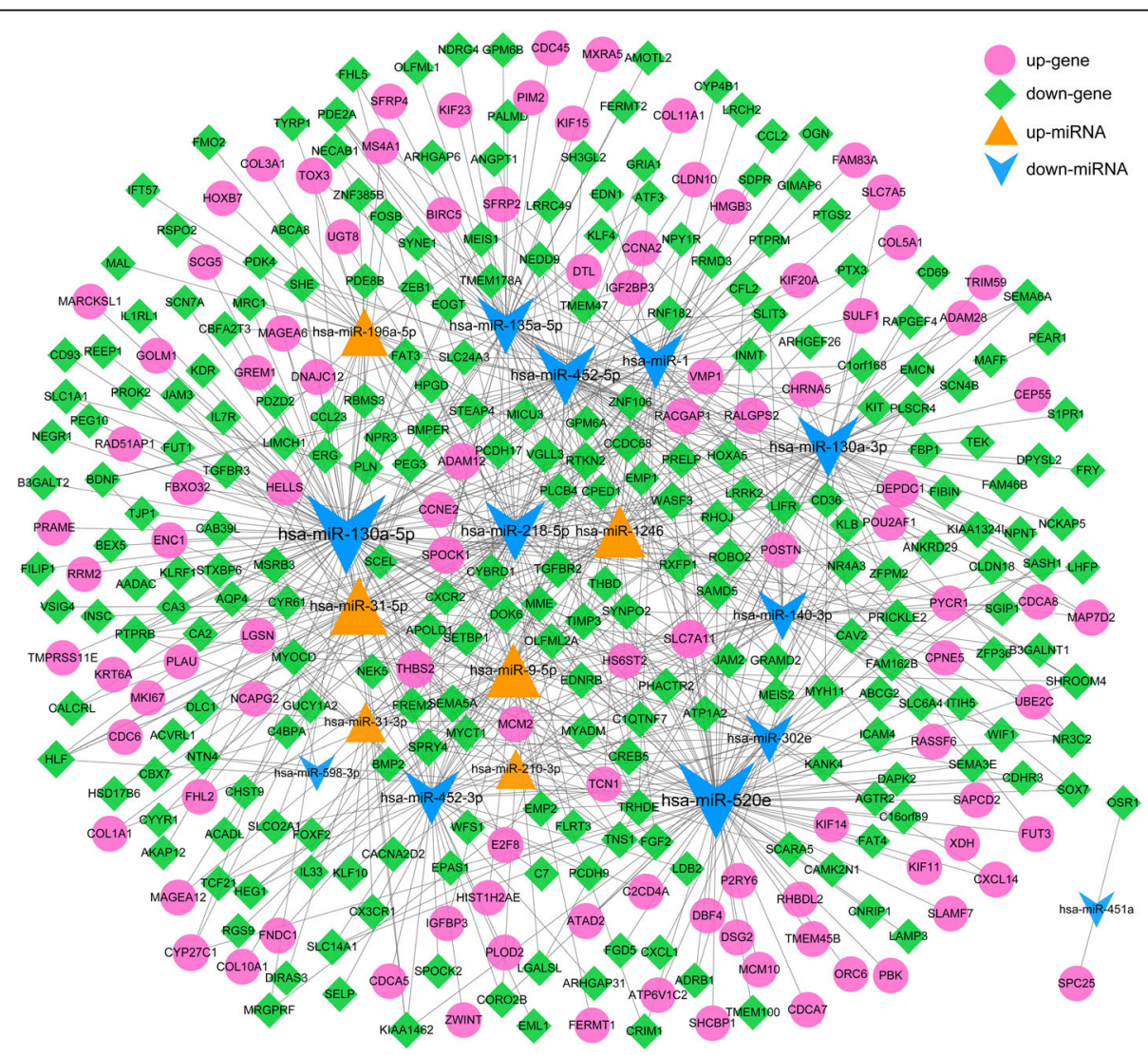

Fig. 3 DEmiR-DEG regulatory network. Yellow trianglesrepresent up-regulated miRNAs, blue arrow indicates down-regulated miRNA, red circles represent up-regulated genes, and green prisms represent down-regulated genes 
and transcriptional misregulation in cancer were significantly enriched by several miRNAs, such as hsa-miR-9-5p, hsa-miR-31-3p, hsa-miR-218-5p and hsa-miR-196a-5p. In addition, KEGG pathways enriched by up- and downregulated genes (Fig. 2b and c) showed that up-regulated genes were significantly enriched in 6 KEGG pathways (cell cycle, protein digestion and absorption, ECMreceptor interaction, focal adhesion, amoebiasis, and Platelet activation) and down-regulated genes were significantly enriched in 6 KEGG pathways (adrenergic signaling in cardiomyocytes, axon guidance, cGMP-PKG signaling pathway, adherens junction, cAMP signaling pathway, and cocaine addiction).

\section{MiRNA-gene regulatory network construction}

The DEmiR-DEG regulation network was shown in Fig. 3, consisting of 364 nodes and 715 interactions. The nodes with high topological score can be regarded as key network nodes. The top 5 miRNAs with higher degree included hsa-miR-130a-5p (down-regulated, degree = 93), hsa-miR-520e (down-regulated, degree $=90$ ), hsa$\mathrm{miR}-452-5 \mathrm{p}$ (down-regulated, degree $=56$ ), hsa-miR130a-3p (down-regulated, degree $=55$ ), hsa-miR-218-5p (down-regulated, degree $=53$ ). The top 5 genes with higher degree included Transforming Growth Factor Beta Receptor $2(T G F B R 2$, down-regulated, degree $=8$ ), Rhotekin $2(R T K N 2$, down-regulated, degree $=7$ ), Solute
Carrier Family 7 Member 11 (SLC7A11, up-regulated, degree =7), Slit Guidance Ligand 3 (SLIT3, downregulated, degree $=7)$, and Semaphorin 5A $(S E M A 5 A$, down-regulated, degree $=7$ ).

\section{Construction of PPI network}

The PPI network of genes involved in the DEmiR-DEG interaction was constructed as Fig. 4, including 860 interactions and 215 nodes (68 up- and 147 down-regulated genes). The top 10 genes with higher degree were as follows: Baculoviral IAP Repeat Containing 5 (BIRC5, upregulated, degree $=36)$, Fibroblast Growth Factor $2(F G F 2$, down-regulated, degree $=34$ ), Rac GTPase Activating Protein $1(R A C G A P 1$, down-regulated, degree $=33)$, Cell Division Cycle 6 (CDC6, up-regulated, degree $=33)$, PDZ Binding Kinase $(P B K$, up-regulated, degree $=32)$, ZW10 Interacting Kinetochore Protein (ZWINT, up-regulated, degree $=32$ ), Kinesin Family Member 11 (KIF11, upregulated, degree $=32$ ), Kinesin Family Member 23 (KIF23, up-regulated, degree $=32)$, Cyclin A2, $(C C N A 2$, upregulated, degree $=32$ ), and Ribonucleotide Reductase Regulatory Subunit M2 (RRM2, up-regulated, degree $=32$ ).

\section{ceRNA network construction}

The lncRNAs were predicted for all 18 miRNAs in the regulatory network, resulting in 9 miRNA-lncRNA relationships, including 5 miRNAs and 7 lncRNAs. We

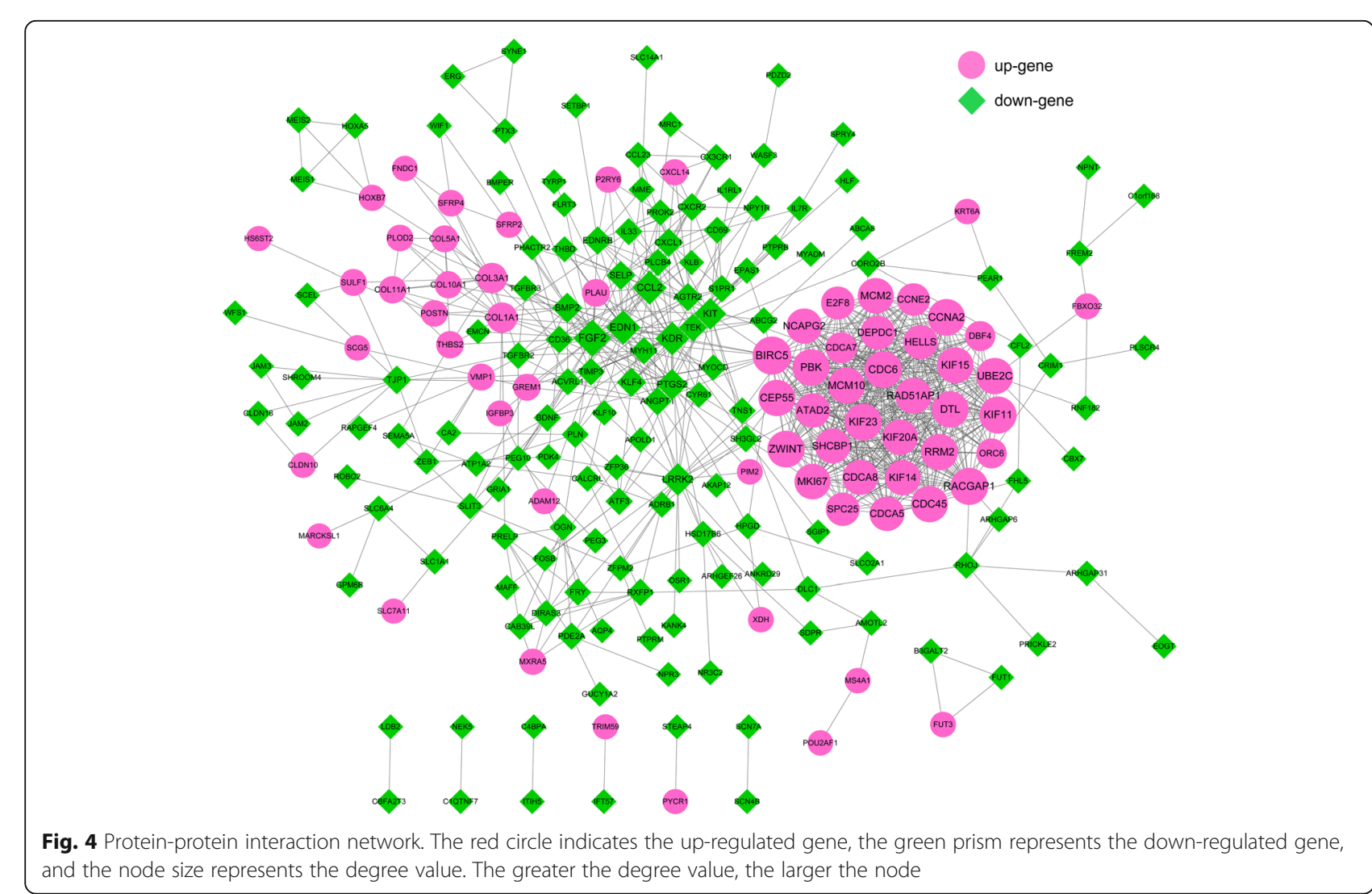


compared these 5 miRNAs in the regulation relationship of DEmiR-DEGs and integrated with miRNA-IncRNAs interaction. A total of 214 relationships were obtained, including 3 up-regulated miRNAs (hsa-miR-9-5p, has-miR-196a-5p, and hsa-miR-31-5p), 2 down-regulated miRNAs (hsa-miR135a-5p and hsa-miR-1), 41 up-regulated genes, 124 downregulated miRNAs, and 7 lncRNAs. The ceRNA network was shown in Fig. 5. The genes RTKN2 and SLIT3 had relative higher degree of 4 and 3, respectively. Both of RTKN2 and SLIT3 can be regulated by hsa-miR-1, hsa-miR-9-5p, and hsa-miR-135a-5p.

\section{Discussion}

In this study, we integrated mRNA expression profile and miRNA expression profile to evaluate changes of gene and miRNA expression in NSCLC. A total of 123
DEmiRs (5 up- and 118 down-regulated miRNAs) and 924 DEGs (309 up- and 615 down-regulated genes) were found. These genes and miRNAs were significantly involved in different pathways including relaxin signaling pathway, adherens junction, and axon guidance. HsamiR-9-5p, has-miR-196a-5p and hsa-miR-31-5p, as well as hsa-miR-1, hsa-miR-218-5p and hsa-miR-135a-5p were shown to have higher degree in the miRNA-gene regulatory network and ceRNA network, respectively. Furthermore, BIRC5 and FGF2, as well as RTKN2 and SLIT3were hubs in the PPI network and ceRNA network, respectively.

Evidence suggests that relaxin, a peptide hormone, can promote invasiveness of breast cancer cell lines through increasing the expression of matrix metalloproteinases (MMPs) [24, 25]. A study has shown that mouse relaxin

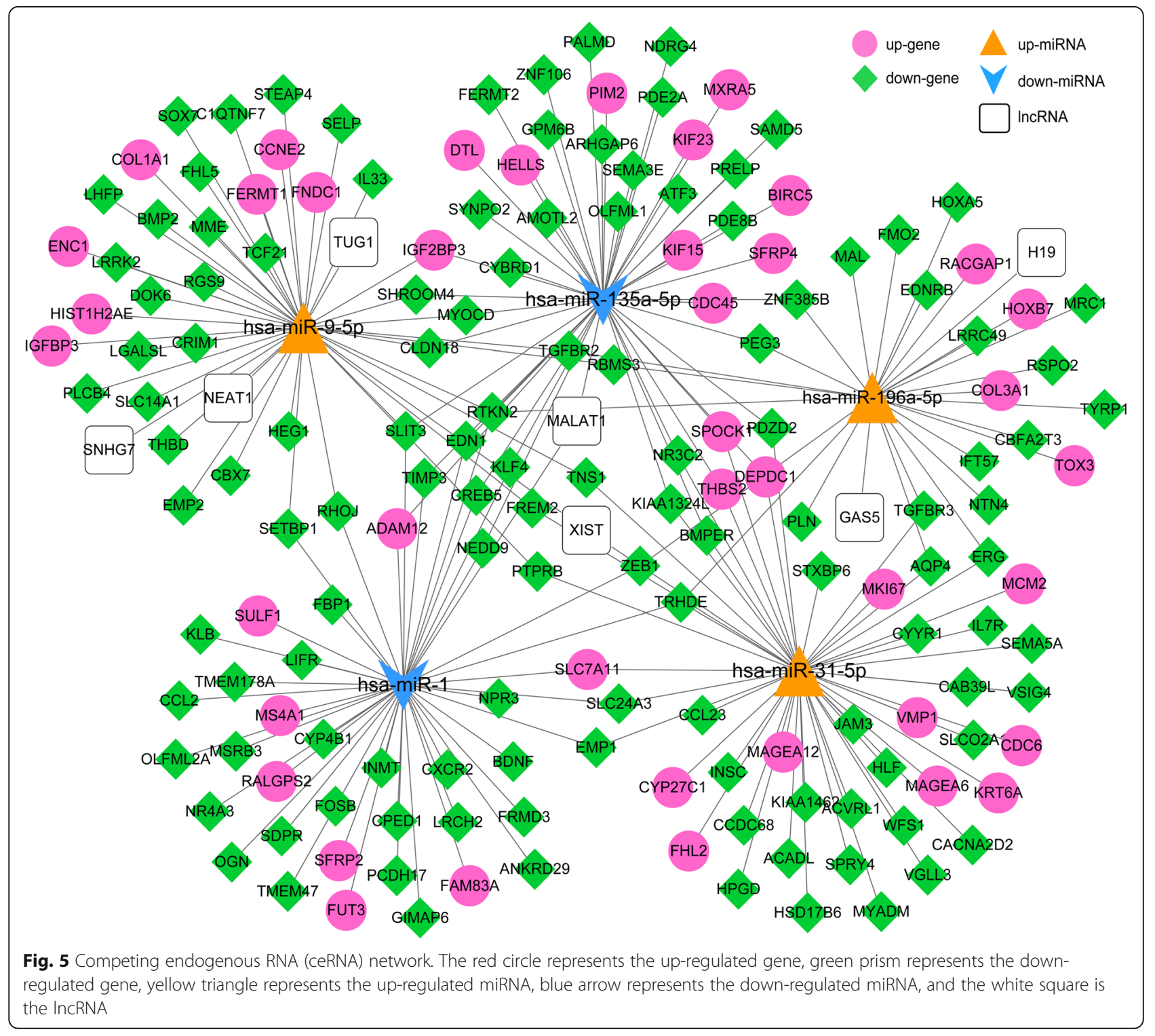


gene expression in the lungs can result in enhanced MMP activity [26]. However, there are few direct evidence targeting the role of relaxin in the progression of lung cancer. In this study, we found that the DEGs and DEmiR were associated with relaxin signaling pathway. Disruption of adherens junction has been demonstrated to play a causal role in cancer initiation and progression [27]. In addition, Notch1 destabilizates the adherens junction complex and influence NSCLC cell proliferation [28]. Cancer cells secrete axon guidance molecules control the invasion and migration of cancer [29]. In this study, we found that DEGs and DEmiRs were also involved in the adherens junction and axon guidance. Taken together, we suggested that relaxin signaling pathway, adherens junction, and axon guidance may play important roles in the pathogenesis of NSCLC.

In addition, we found several miRNAs had higher degrees in the constructed network, including hsa-miR-95p, has-miR-196a-5p, hsa-miR-31-5p, hsa-miR-1, hsamiR-218-5p, and hsa-miR-135a-5p. Xu et al. reported up-regulation of miR-9 as poor prognostic biomarker in NSCLC patients [30]. A study showed that miR-196a could promote cell proliferation an invasion of NSCLC through regulating HOXA5 [31]. miR-31 acts as an oncogenic miRNA in lung cancer [32]. Moreover, there are evidence between the interaction of miR-1, miR-218, and miR-135a and lung cancer [33-35], which are in line with our study. Thus, we suggest the significant roles of hsa-miR-9-5p, has-miR-196a-5p, hsa-miR-31-5p, hsa-miR1, hsa-miR-218-5p, and hsa-miR-135a-5p in NSCLC progression, which may be effective biomarkers used for the cancer diagnosis and therapy.

Furthermore, BIRC5 and FGF2, as well as RTKN2 and SLIT3 were hubs in the PPI network and ceRNA network. Previous study showed that BIRC5 was up-regulated in NSCLC [36]. FGF2-mediated autocrine signaling is activated in NSCLC cell lines [37]. Epigenetic inactivation of SLIT3 has been found in human cancers [38]. However, there is no evidence regarding to the role of RTKN2 in lung cancer. Nevertheless, we suggested that BIRC5, FGF2, RTKN2 and SLIT3 may be important for lung cancer; but experiment verification should be performed in future.

\section{Conclusion}

In conclusion, the present study has demonstrated the potentially critical roles of several pathways (adherens junction, relaxin signaling pathway and axon guidance) miRNAs (hsa-miR-9-5p, has-miR-196a-5p, hsa-miR-315p, hsa-miR-1, hsa-miR-218-5p, and hsa-miR-135a-5p) and genes (BIRC5, FGF2, RTKN2 and SLIT3) in the progression of NSCLC. The miRNAs and genes may be favorable biomarkers for patients with this cancer. However, there were still some limitations. Firstly, not all
mRNA samples were from miRNA samples, which will reduce the statistical power for examining the relationship between miRNA and mRNA. Secondly, this findings were identified in one cohort, which should be confirmed in other cohorts. And hence future validations with the miRNA and mRNA samples from the same patients should be conducted.

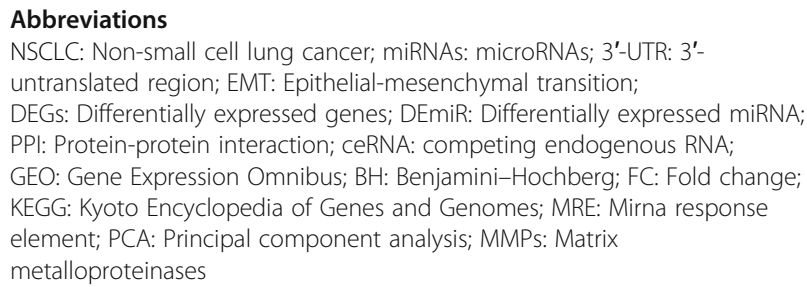

Acknowledgements

Not applicable.

\section{Authors' contributions}

Conception of the research and drafting the manuscript: XJ; Acquisition of data: HW; Analysis and interpretation of data: YG and ZZ; Draft the manuscript: XJ. All authors have read and approved the manuscript.

\section{Funding}

Not applicable.

Availability of data and materials

In this study, miRNA and mRNA microarray datasets were downloaded from the GEO database under the accession number GSE102286 and GSE101929, respectively.

Ethics approval and consent to participate

Not applicable.

Consent for publication

Not applicable.

\section{Competing interests}

The authors declare that they have no competing interests.

\section{Author details}

'Department of Respiration, The First Hospital of Jilin University, No. 1 Xinminda Street, Changchun 130021, China. ${ }^{2}$ PICU, The First Hospital of Jilin University, Changchun 130021, China. ${ }^{3}$ Department of Nephrology, The First Hospital of Jilin University, Changchun 130021, China.

Received: 1 November 2019 Accepted: 5 April 2020

Published online: 16 April 2020

\section{References}

1. Xu F, Xu P, Cui W, Gong W, Wei Y, Liu B, et al. Neutrophil-to-lymphocyte and platelet-to-lymphocyte ratios may aid in identifying patients with nonsmall cell lung cancer and predicting tumor-node-metastasis stages. Oncol Lett. 2018;16(1):483-90.

2. Chen D, Zhang L, Huang J, Liu K, Chuai Z, Yang Z, et al. BRAF mutations in patients with non-small cell lung cancer: a systematic review and metaanalysis. PLoS One. 2014;9(6):e101354

3. Li R, Fan X, Duan F, Jiang Z, Pan H, Luo L, et al. Proscillaridin a induces apoptosis and suppresses non-small-cell lung cancer tumor growth via calcium-induced DR4 upregulation. Cell Death Dis. 2018;9(6):696.

4. Riveiro ME, Kwee I, Astorguesxerri L, Bekradda M, Vazquez R, Rinaldi A, et al. Abstract 3530: gene expression profile of OTX015, a BET bromodomain inhibitor, in preclinical models of non-small-cell lung cancer (NSCLC) and small-cell lung cancer (SCLC) models. Cancer Res. 2015;75(15 Supplement):3530. 
5. Li G, Gao Y, Cui Y, Zhang T, Cui R, Yang J, et al. Overexpression of CD44 is associated with the occurrence and migration of non-small cell lung cancer. Mol Med Rep. 2016;14(4):3159-67.

6. Altenberger C, Heller G, Ziegler B, Tomasich E, Marhold M, Topakian T, et al. SPAG6 and L1TD1 are transcriptionally regulated by DNA methylation in non-small cell lung cancers. Mol Cancer. 2017;16(1):1.

7. Morris S, Vachani A, Pass HI, Rom WN, Ryden K, Weiss GJ. Whole blood FPR1 mRNA expression predicts both non-small cell and small cell lung cancer. Int J Cancer. 2018;142(11):2355-62.

8. Yu AM, Tian Y, Tu MJ, Ho PY, Jilek JL. MicroRNA Pharmacoepigenetics: posttranscriptional regulation mechanisms behind variable drug disposition and strategy to develop more effective therapy. Drug Metab Dispos. 2015: 44(3):308.

9. Hosseini SM, Soltani BM, Tavallaei M, Mowla SJ, Tafsiri E, Bagheri A, et al. Clinically significant dysregulation of hsa-miR-30d-5p and hsa-let-7b expression in patients with surgically resected non-small cell lung cancer. Avicenna J Med Biotechnol. 2018;10(2):98-104.

10. Yang F, Wei K, Qin Z, Liu W, Shao C, Wang C, et al. MiR-598 suppresses invasion and migration by negative regulation of Derlin-1 and epithelialmesenchymal transition in non-small cell lung cancer. Cell Physiol Biochem. 2018:47(1):245-56.

11. Olivieri F, Capri M, Bonafe M, Morsiani C, Jung HJ, Spazzafumo L, et al. Circulating miRNAs and miRNA shuttles as biomarkers: perspective trajectories of healthy and unhealthy aging. Mech Ageing Dev. 2017;165(Pt B):162-70.

12. Huang R, Gao L. Identification of potential diagnostic and prognostic biomarkers in non-small cell lung cancer based on microarray data. Oncol Lett. 2018;15(5):6436-42

13. Clough $\mathrm{E}$, Barrett T. The gene expression omnibus database. Methods Mol Biol. 2016;1418:93-110.

14. Waggott D, Chu K, Yin S, Wouters BG, Liu F-F, Boutros PC. NanoStringNorm: an extensible $R$ package for the pre-processing of NanoString mRNA and miRNA data. Bioinformatics. 2012;28(11):1546-8.

15. Ritchie ME, Phipson B, Wu D, Hu Y, Law CW, Shi W, et al. Limma powers differential expression analyses for RNA-sequencing and microarray studies. Nucleic Acids Res. 2015;43(7):e47.

16. Dweep H, Gretz N. miRWalk2. 0: a comprehensive atlas of microRNA-target interactions. Nat Methods. 2015;12(8):697.

17. Kanehisa M, Goto S. KEGG: Kyoto encyclopedia of genes and genomes. Nucleic Acids Res. 2000;28(1):27-30

18. Yu G, Wang L-G, Han Y, He Q-Y. clusterProfiler: an R package for comparing biological themes among gene clusters. OMICS. 2012;16(5):284-7.

19. Shannon P, Markiel A, Ozier O, Baliga NS, Wang JT, Ramage D, et al. Cytoscape: a software environment for integrated models of biomolecular interaction networks. Genome Res. 2003;13(11):2498-504.

20. Szklarczyk D, Franceschini A, Kuhn M, Simonovic M, Roth A, Minguez $P$, et al. The STRING database in 2011: functional interaction networks of proteins, globally integrated and scored. Nucleic Acids Res. 2010;39(suppl_ 1):D561-8.

21. Von Mering C, Jensen LJ, Snel B, Hooper SD, Krupp M, Foglierini M, et al. STRING: known and predicted protein-protein associations, integrated and transferred across organisms. Nucleic Acids Res. 2005;33(suppl_1):D433-7.

22. Ala U, Karreth FA, Bosia C, Pagnani A, Taulli R, Léopold V, et al. Integrated transcriptional and competitive endogenous RNA networks are crossregulated in permissive molecular environments. Proc Natl Acad Sci. 2013: 110(18):7154-9.

23. Li J-H, Liu S, Zhou H, Qu L-H, Yang J-H. starBase v2. 0: decoding miRNAceRNA, miRNA-ncRNA and protein-RNA interaction networks from largescale CLIP-Seq data. Nucleic Acids Res. 2013;42(D1):D92-7.

24. Binder C, Hagemann T, Husen B, Schulz M, Einspanier A. Relaxin enhances in-vitro invasiveness of breast cancer cell lines by up-regulation of matrix metalloproteases. Mol Hum Reprod. 2002;8(9):789-96.

25. Bani D. Relaxin: a pleiotropic hormone. Gen Pharmacol. 1997;28(1):13-22.

26. Silvertown JD, Walia JS, Summerlee AJ, Medin JA. Functional expression of mouse Relaxin and mouse Relaxin-3 in the lung from an Ebola virus glycoprotein-Pseudotyped lentivirus via tracheal delivery. Endocrinology. 2006;147(8):3797-808.

27. Vasioukhin V. Adherens junctions and cancer. Subcell Biochem. 2012;60: 379-414.

28. Kim A, Kim EY, Cho EN, Kim HJ, Kim SK, Chang J, et al. Notch1 destabilizes the adherens junction complex through upregulation of the snail family of
E-cadherin repressors in non-small cell lung cancer. Oncol Rep. 2013;30(3): 1423-9.

29. Mancino M, Ametller E, Gascón P, Almendro V. The neuronal influence on tumor progression. Biochim Biophys Acta. 2011;1816(2):105-18.

30. Xu T, Liu X, Han L, Shen H, Liu L, Shu Y. Up-regulation of miR-9 expression as a poor prognostic biomarker in patients with non-small cell lung cancer. Clin Transl Oncol. 2014;16(5):469-75.

31. Liu XH, Lu KH, Wang KM, Sun M, Zhang EB, Yang JS, et al. MicroRNA-196a promotes non-small cell lung cancer cell proliferation and invasion through targeting HOXA5. BMC Cancer. 2012;12(1):348.

32. Liu X, Sempere LF, Ouyang H, Memoli VA, Andrew AS, Luo Y, et al. MicroRNA-31 functions as an oncogenic microRNA in mouse and human lung cancer cells by repressing specific tumor suppressors. J Clin Invest. 2010;120(4):1298-309.

33. Nasser MW, Datta J, Nuovo G, Kutay H, Motiwala T, Majumder S, et al. Down-regulation of micro-RNA-1 (miR-1) in lung cancer. J Biol Chem. 2008; 283(48):33394-405.

34. Wu DW, Cheng YW, Wang J, Chen CY, Lee H. Paxillin predicts survival and relapse in non-small cell lung cancer by MicroRNA-218 targeting. Cancer Res. 2010;70(24):10392-401.

35. Shi H, Ji Y, Zhang D, Liu Y, Fang P. MiR-135a inhibits migration and invasion and regulates EMT-related marker genes by targeting KLF8 in lung cancer cells. Biochem Biophys Res Commun. 2015;465(1):125-30.

36. Văshlia NA, Zinov'eva MV, Sass AV, Kopantsev EP, Vinogradova TV, Sverdlov ED. Increase of BIRC5 gene expression in non-small cell lung cancer and esophageal squamous cell carcinoma does not correlate with expression of genes SMAC/DIABLO and PML encoding its inhibitors. Mol Biol. 2008;42(4):652.

37. Marek L, Ware KE, Fritzsche A, Hercule P, Helton WR, Smith JE, et al. Fibroblast growth factor (FGF) and FGF receptor-mediated autocrine signaling in nonsmall-cell lung cancer cells. Mol Pharmacol. 2009;75(1):196-207.

38. Dickinson RE, Dallol A, Bieche I, Krex D, Morton D, Maher ER, et al. Epigenetic inactivation of SLIT3 and SLIT1 genes in human cancers. Br J Cancer. 2004;91(12):2071-8.

\section{Publisher's Note}

Springer Nature remains neutral with regard to jurisdictional claims in published maps and institutional affiliations.
Ready to submit your research? Choose BMC and benefit from:

- fast, convenient online submission

- thorough peer review by experienced researchers in your field

- rapid publication on acceptance

- support for research data, including large and complex data types

- gold Open Access which fosters wider collaboration and increased citations

- maximum visibility for your research: over $100 \mathrm{M}$ website views per year

At BMC, research is always in progress.

Learn more biomedcentral.com/submissions 